\title{
The Inferior Pectoral Artery, A New Definition
}

\author{
By
Kodo KODAMA, Munesato YAMADA, Katsushi KAWAI, Keishi OKAMOTO and Shigeki MIZUKAMI*

\author{
Department of Anatomy, Kanazawa University School of Medicine, \\ 13-1 Takaramachi, Kanazawa, 920, and \\ Department of Anatomy, School of Nursing, Fukui Prefectural College* \\ Oh-hatamachi, Fukui 910, Japan
}

-Received for Publication, December 12, 1986-

\begin{abstract}
Key words: A pectoralis inferior, M. pectoralis major, A. axillaris, A. brachialis superficialis, variation

Summary. The pectoralis major muscle receives its arterial supply from the A. thoracoacromialis, by its pectoral branch. But, the "terminal branch" entering the lowest neurovascular hilum of the muscle is usually separate and arises from the axillary artery at different levels, either independently or in common with other arteries such as $\mathbf{A}$. thoracoacromialis or $\mathbf{A}$. thoracica lateralis. Distally, the artery may send off various superficial branches, including the A. brachialis superficialis superior. The A. pectoralis inferior may be better described in these instances as to ramify as its first offshoot, especially if the last mentioned artery is well developed. Besides, the A. pectoralis inferior never ramifies from the deep artery of the arm. It has been verified, therefore, that the two arteries are interpreted identical to each other, and the A. pectoralis inferior should be regarded morphologically significant. It deserves a new definition.
\end{abstract}

In a review on the axillary artery dealing theoretically with the patterns of ramification, Yamada (1967) has given the first mention to the A. pectoralis inferior with an insight that this artery should have certain morphogenetic significance. However, as his primary purpose was then directed mainly to a discussion of what he proposed as the $\mathbf{A}$. subscapularis superficialis, he could not enter the details. It is, therefore, intended in the present paper to verify the A. pectoralis inferior, or "API" to make it short, on a more systematic basis and a number of examples.

\section{Materials and Methods}

Pertaining to the proposed API, the human axillary artery has been critically surveyed together with ramification during six academic years, 1980-1985, at the Kanazawa University School of Medicine.

Altogether 179 Japanese subjects, totalling 355 sides, have been examined. Special attention has been paid to the topographical relations of the arteries to the brachial plexus and its branches, but intentionally little attention has been paid to the conventional terms or definitions with a fear that these may spoil the whole endeavour. Arbitrary definitions have to be set out(vide Appendix: Glossary), and verification has 
been made as to the pertinence of every branch. The findings were analysed on the line of "typological anatomy" after Nishi (1961), and the recording were made out by every available means, such as manual depiction, photography either monocular (routine) or binocular (stereo), or by video where it is worth.

\section{Findings}

\section{Verification of the standard description:}

The branch called $\mathbf{R}$. pectoralis of the A. thoracoacromialis is described in the standard textbooks, for example the most up-todate 36th edition of Gray (Williams and Warwick, 1980), to "descend between the two pectoral muscles and is distributed to them and to the breast, anastomosing with the intercostal branches of the internal thoracic artery and with the lateral thoracic artery". This branch should be interpreted muscular in nature because its terminal branches are distributed mainly to the pectoralis major with a small contribution to the pectoralis minor (Fig. 2). If, however, attention is paid to the lowest neurovascular hilum, in which the artery terminates, such examples occur in 27 sides only (7.6\%). In the remaining majority of cases, that is to say, in 328 sides (ca. 92\%), the same lowest neurovascular hilum receives separately another independent branch either from the axillary artery or from one of its anterior branches. Such is an artery which was originally proposed by Yamada as A. pectoralis inferior, or API.

2. A. pectoralis inferior (API) as examined by the authors:

Figs. 3a and $3 \mathrm{~b}$ show practically all the important varieties of the observed API in

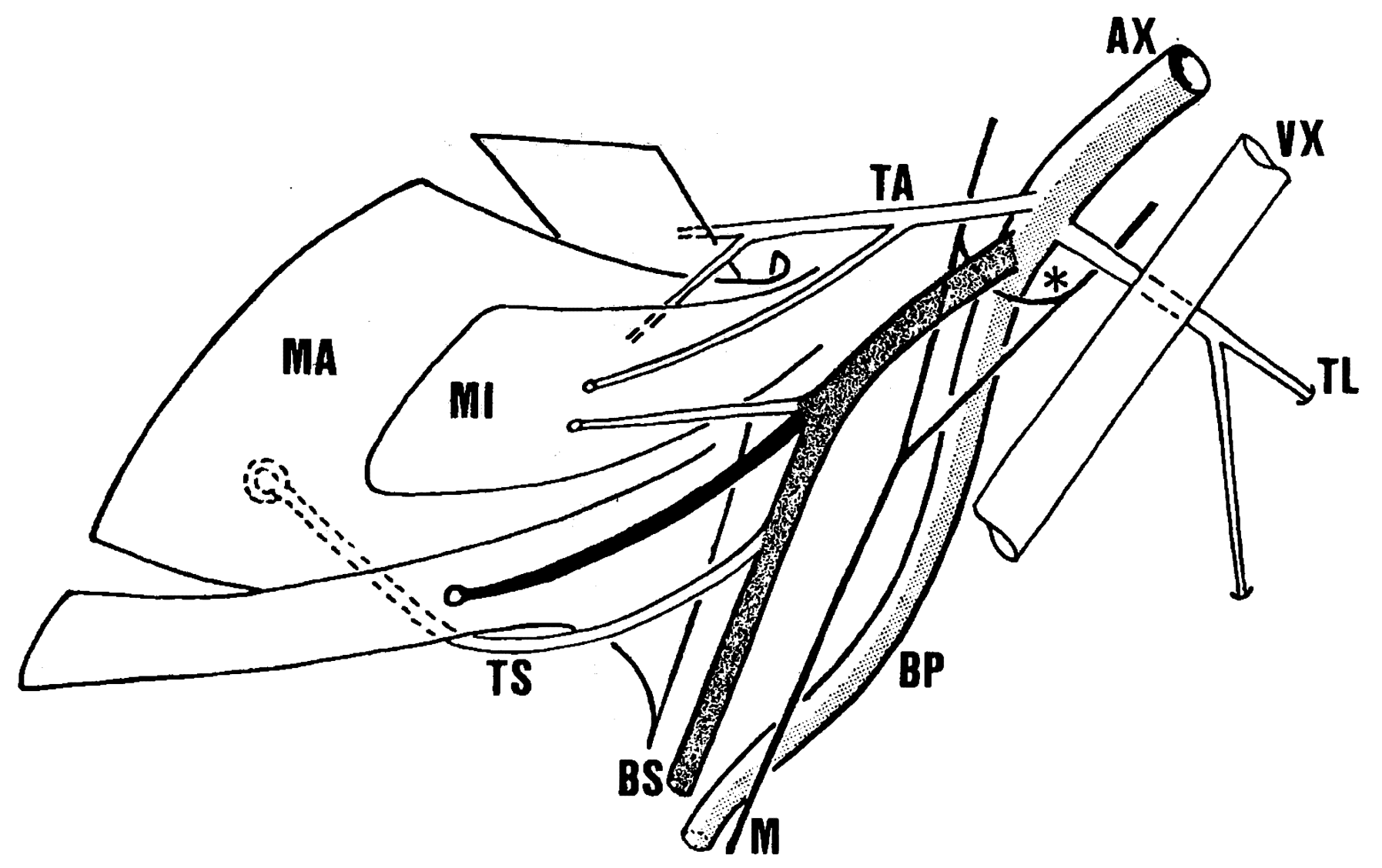

Fig. 1. Identification Key to Figures. AX: A. axillaris, BP: A. brachialis (profunda), BS: A. brachialis superficialis (superior), $M: N$. medianus, MA: $M$. pectoralis major, MI: $M$. pectoralis minor, TA: A. thoracoacromialis, TL: A. thoracica lateralis, TS: A. thoracica superficialis with mamma at the end, VX: V. axillaris, * Ansa pectoralis. A. pectoralis inferior (API) is shown black. 

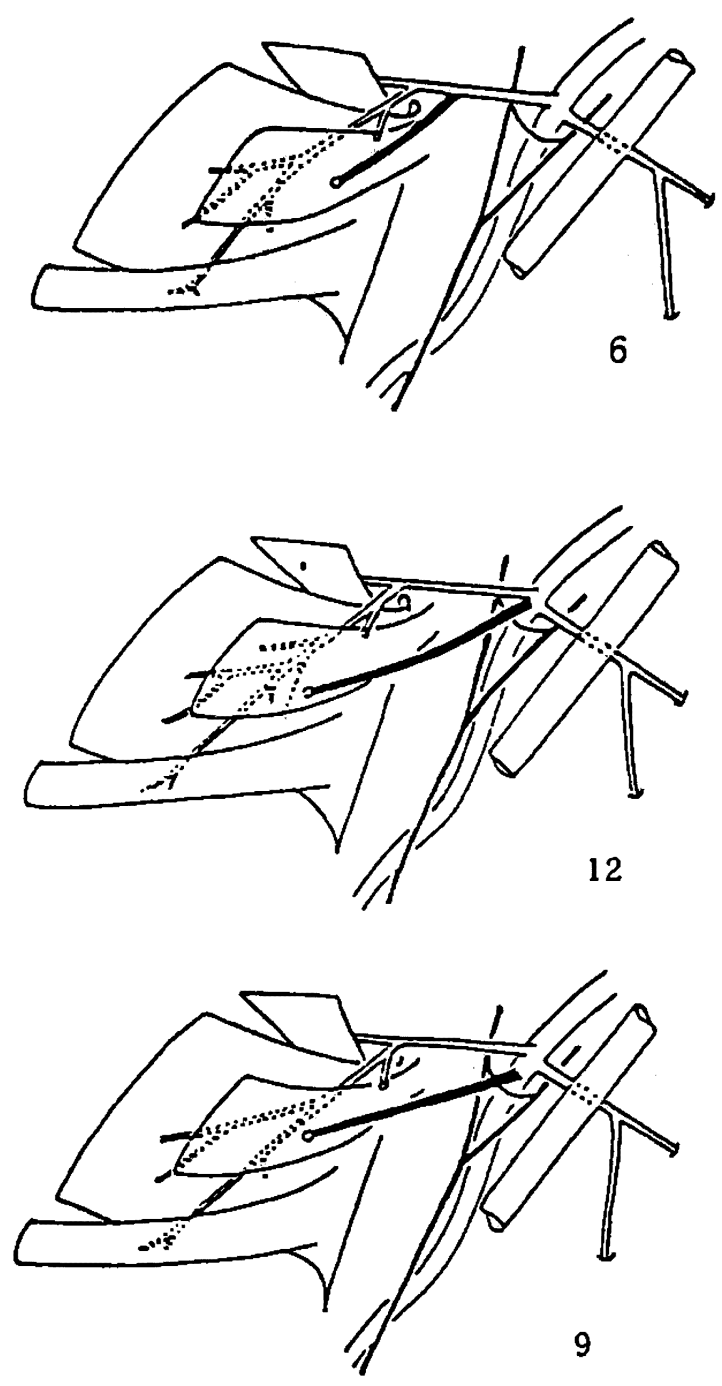

Fig. 2. Arteries of the pectoralis muscles, in the pattern of textbook standard. For identification, refer to Fig. 1. A. thoracoacromialis (TA) supplies the pectoralis major (MA) by its $R$. pectoralis to as far as the lowest neurovascular hilum. This terminal branch is usually taken over by one of the anterior branches of the axillary artery (AX), which is proposed in this paper as $A$. pectoralis inferior (API). Twigs shown black are interpreted to be its precursor, see text. Figures show number of examples.

a logical series and synoptic diagram, with frequency of each division. However, as there are inevitably some exceptions (quod vide) which are better excluded from this diagram, Figs. 4 and 5 should be referred to also.
(2-1) An independent branch to originate directly from the axillary artery:

The typical APIs are present as frequently as 232 among 355 sides examined (65.4\%). These are tabulated in Figs. $3 a$ and $3 b$ in divisions $\mathrm{A} / 1, \mathrm{~A} / 4, \mathrm{~A} / 5, \mathrm{~B} / 1, \mathrm{~B} / 4, \mathrm{~B} / 5$, $\mathrm{C} / 1, \mathrm{C} / 4$ and $\mathrm{C} / 5$. They may be divided roughly into three major categories, according to origin and relation to the Ansa pectoralis (simply as Ansa hereafter) as follows:

A) Arteries of the first category, which arise proximally from the second part of the axillary artery and pass in front of the Ansa, are observed in $169 / 355$ sides (47.6\%). This is the most frequent of all $(\mathrm{A} / 1, \mathrm{~B} / 1$ and $\mathrm{C} / 1$ ).

B) Next comes the second or intermediate cateogry ( 31 sides; $8.7 \%$ ), in which the artery in question arises, as in the previous category, from the second part of the axillary artery. This differs, however, in one respect that it penetrates the Ansa itself (Series 4).

C) Lastly come the distal ones (53 sides; 14.9\%), which originate (always independently!) from the axillary artery and pass between the ansa and the median nerve (Ser. 5).

In all the categories mentioned above, definite stages of developments may be noticed, which comprise: 1) the API proper, or the artery which does not issue side branch at all (Category A); 2) the ones which issue a fine muscular twig to the pectoralis minor (Category B); and 3) the ones which send off the $A$. thoracica superficialis (or R. mammarius lateralis, PNA) in addition (Category $\mathrm{C}$ ).

(2-2) The artery which arises from one of the neibouring arteries:

The API may originate in common with, or from, either the $A$. thoracoacromialis (36 sides, $10.1 \% ; \mathrm{A} / 2$ ), or the $\mathrm{A}$. thoracia lateralis (19 sides, $5.4 \% ; \mathrm{A} / 3, \mathrm{~B} / 3, \mathrm{C} / 3$ and $F / 3)$. The latter artery includes its 

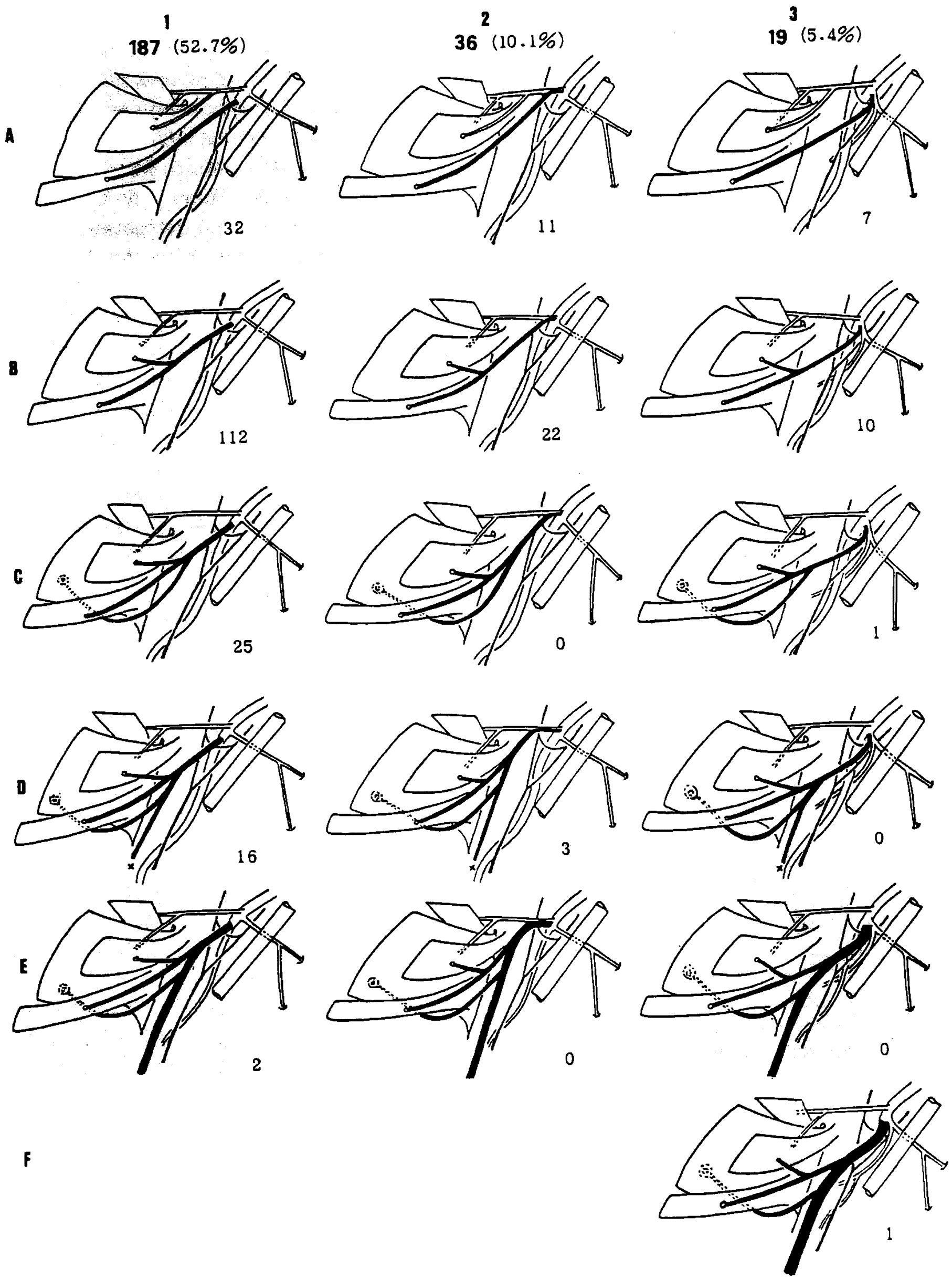

Fig. 3a. Diagram of the typical API, which arises most proximally, to show its various dispositions in tabular form (to be continued to Fig. 3b). For identification, refer to Fig. 1. Categories A-F are arranged according to grades of development, and Series 1-3 in tiers according to origins. Number of each example is shown by figures. 
4

$31(8.7 \%)$

5

$53(14.9 \%)$
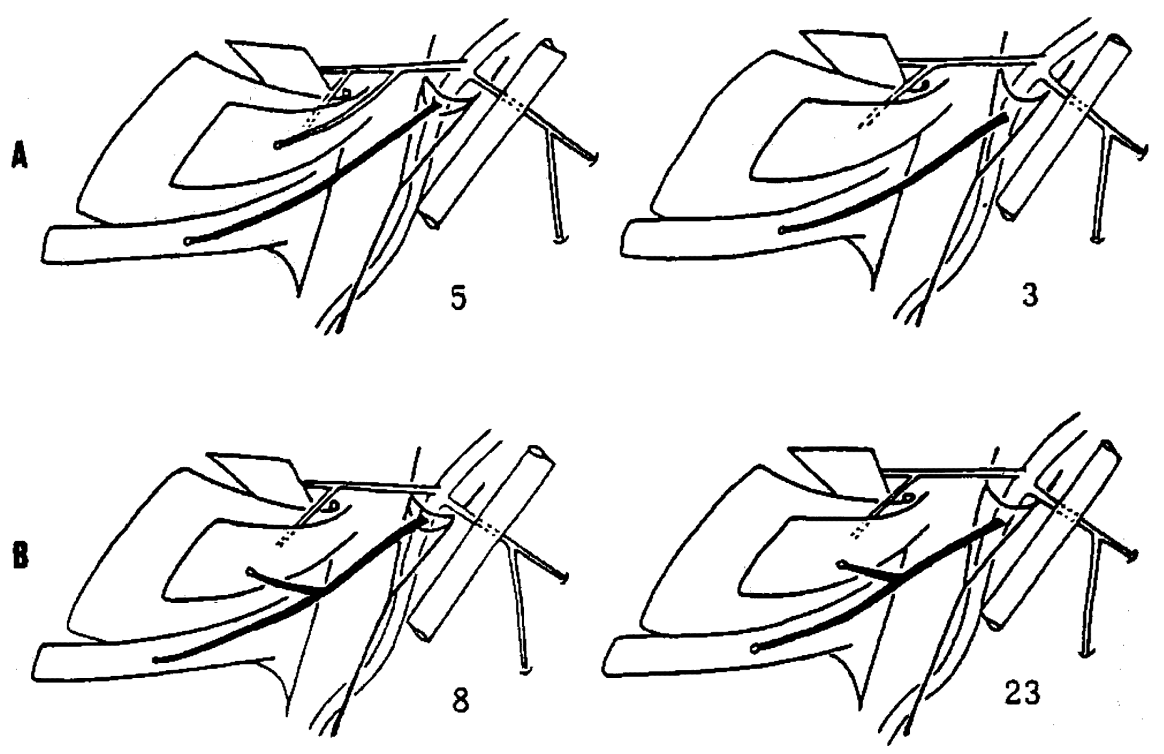

6
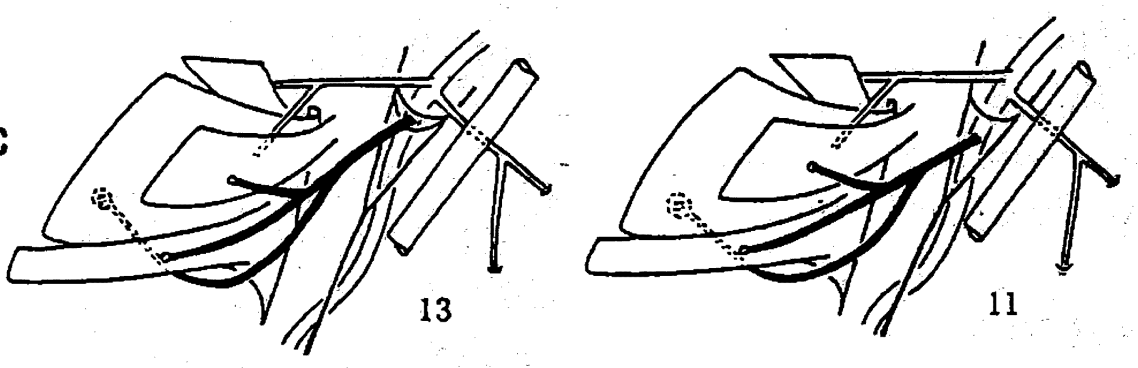

D
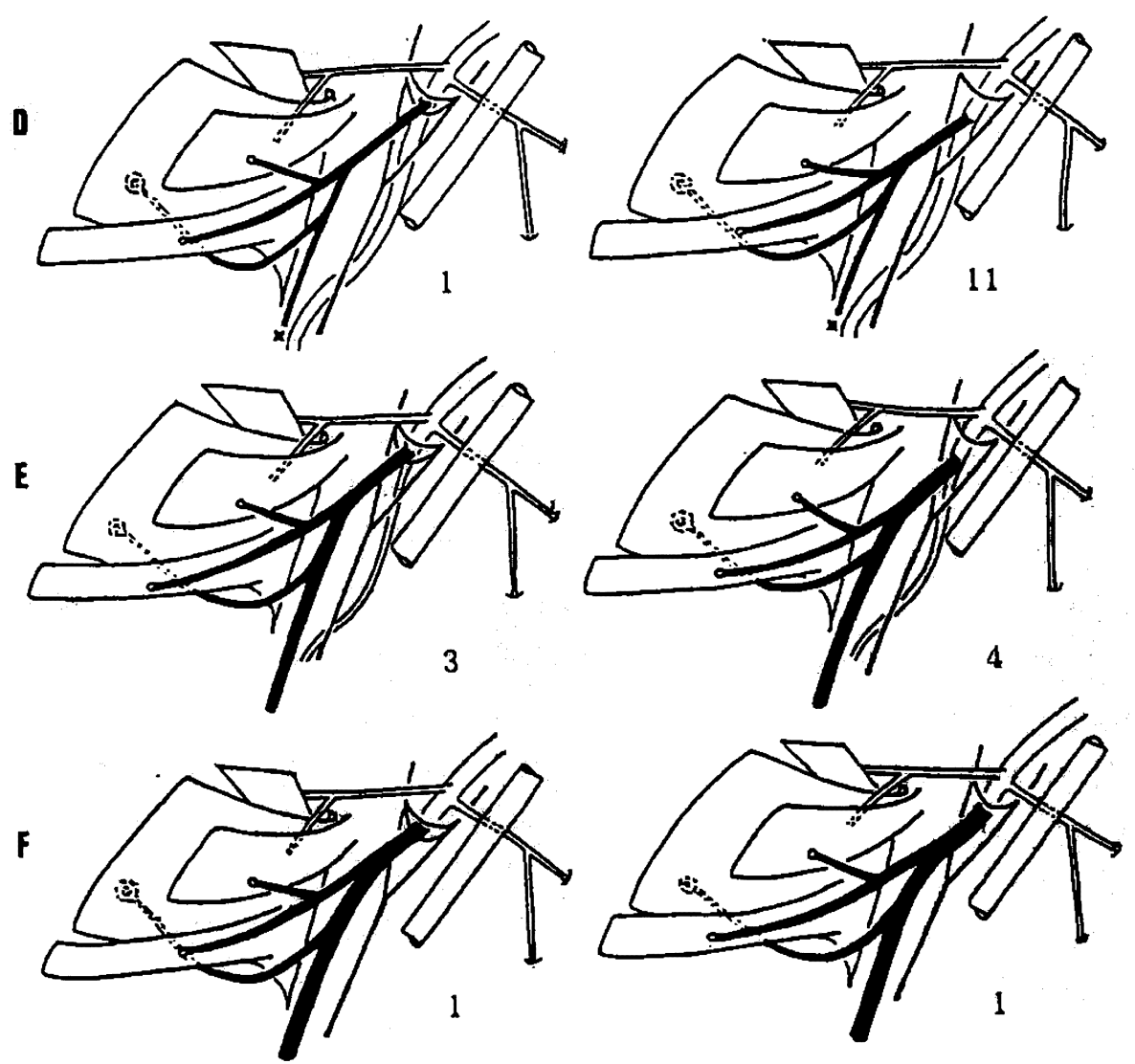

Fig. 3b. Diagram of the typical API, which arises more distally, to show its various dispositions (continued form Fig. 3a). For identification, refer to Fig. 1. Categories A-F are the same as Fig. 3a, but Series 4 and 5 differ in levels of origin. Number of each example is shown by figures. 
affiliated members such as $R$. subscapularis superficialis (two sides), A. thoracodorsalis superficialis (two sides) or A. subscapularis superficialis (15 sides), even if these are not specified in Fig. 3a (vide Appendix: Glossary).

At any rate, a remark may be necessary pertaining to $F / 3$ in particular, because it is fairly special. In it, the cords of the brachial plexus do not surround the axillary artery as in the standard disposition, or in the foregoing divisions $A / 3$ to $C / 3$. In other words, the axillary artery passes below the medial cord of the plexus, taking the course of the A. subscapularis superficialis and continues in this way to as far as the $\mathbf{A}$. profunda brachii. On the other hand, this artery sends off the A. brachialis superficialis superior, or according to the authors' criterion, the A. brachialis superficialis suprema.

Now, one more remark is also needed to $\mathrm{D} / 3$ and $\mathrm{E} / 3$, because these did not actually happen at all, in the present investigation, anyhow. Even so, both are expected with good reasons to happen, even if the probability may be extremely low.

Coming back to the evaluation of this category, it should be noted that the artery follows, as the categories proceed from $A$ to $C$, exactly the same sequence of ramification as in the previous paragraph.

(2-3) Independent artery arising from the $A$. brachialis superficialis:

However, not all the independent API directly ramifies from the axillary artery (11 sides: Categories $\mathrm{E}$ and $\mathrm{F}$, with an exception of $F / 3$ ). The parent artery in these categories is one of the various kinds of the $A$. brachialis superficialis superior, which are to be subdivided according to the same principle as the API itself. Thus, designations of these superficial arteries by the authors are: 1) The most proximal $A$. brachialis superficialis suprema (BSR), which runs in front of the Ansa (E/1 and $F / 1) ; 2$ ) A. brachialis superficialis perforans (BSP), which passes through the Ansa (E/4 and F/4), and 3). Next distal A. brachialis superficialis superior (BSS), which emerges from between the Ansa and that of the median nerve $(E / 5$ and $F / 5)$. In other words, the A. brachialis superficialis, irrespective of differing levels of origins, will ramify as its first offshoot the API. But if by any chance the superficial parent artery happens to share a common trunk of origin with the A. thoracoacromialis (E/2), the API will appear to ramify after the A. thoracoacromialis. Although this kind was not detected among the present findings, it is very likely to happen.
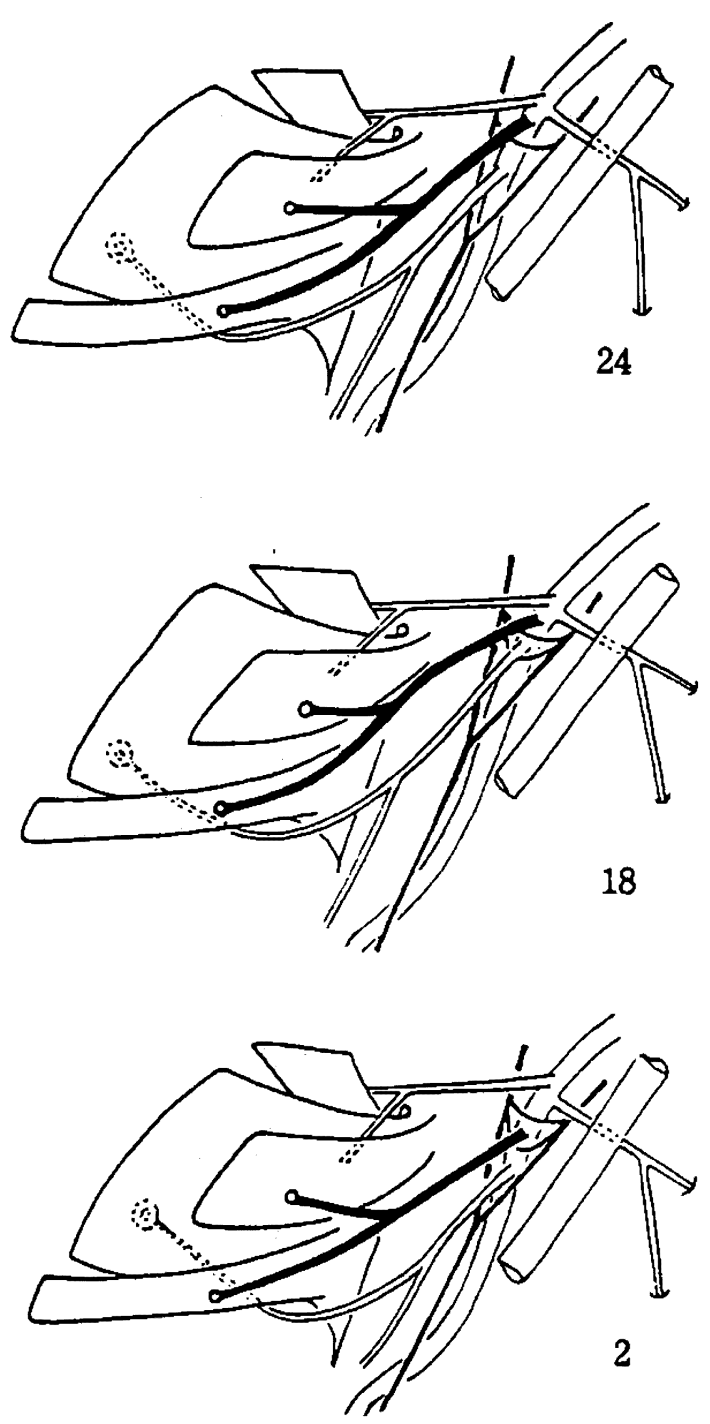

Fig. 4. Segmented API (supplement to Figs. $3 a$ and $3 b$ ). For interpretation, see text. 
It is interesting to note, in this connexion, that the API like Category B is sometimes combined with the separate $\mathbf{A}$. brachialis superficialis superior as its next neibour (Fig. 4). So far as 44 examples (12.4\%) are concerned, the sequence of branching is uniform and is never reversed. And in all the examples, the proximal API and the distal A. brachialis superficialis superior remain separated either by the whole or part of the Ansa in between. This finding would suggest that, otherwise, the API may possibly branch off from this $\mathbf{A}$. brachialis superficialis superior.

(2-4) The artery of various origins, but combined with the less developed $A$. brachialis superficialis, plus $A$. thoracica superficialis (Category D):

One will notice, in the meantime, that the various API of Category D is combined in 31 sides $(8.7 \%)$ with the standard A. brachialis, which submerges distally beneath the median nerve. However, as the API of this category issues also an offshoot of rudimentary $\mathbf{A}$. brachialis superficialis to the arm, which is specified by $x$ in Figs. $3 a$ and $3 b$, the deep A. brachialis itself is, to a certain degree and as the condition may be, substituted by the former, and therefore, becomes more or less weaker. From the point of view of typological anatomy, this category should represent the initial transitional phase to develop to the categories E and F. But as its A. brachialis superficialis is more or less rudimentary, one might possibly overlook or neglect it, even if he happens to come across unpreparedly with such a branch. This is the particular reason why the authors intentionally deals with this category separately.

Whenever the A. brachialis superficialis is combined with the API, it is noteworthy that the latter artery never happens to ramify from the standard $A$. brachialis which lies deep to the median nerve (hence $\mathbf{A}$. brachialis profunda). This fact well indicates that the API represents the superficial layer, and it may be stressed that the $A$. brachialis superficialis shows exactly the same tendency in variation.

(2-5) Oddments of the A. pectoralis inferior: Deviations (Fig. 5): In two instances, the API has been found to ramify from the $A$. thoracica lateralis, but not from its proximity. Instead, these APIs originate from such a distal portion of the $A$. thoracica lateralis after it crosses beneath the axillary vein.

One of these branches off the A. thoracica superficialis (Fig. 5, top), whereas another is combined with the variant $A$. thoracoacromialis, and branches off even the A. brachialis superficialis which is weak (Fig. 5, bottom). This is particularly noteworthy because a superficial trunk artery of the arm may be suggested to develop on this route.

Vestiges: As mentioned in the beginning, the $R$. pectoralis like the textbook standard sends off, in six sides, a small contribution to the deep side of the pectoralis minor (Fig. 2, top). Of greater interest are the
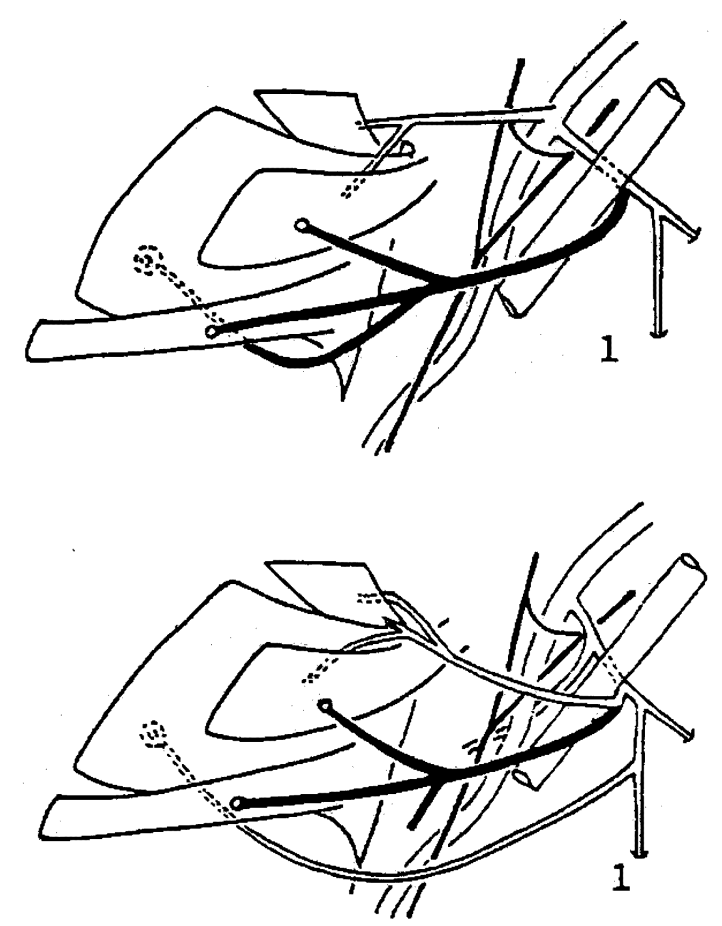

Fig. 5. Aberrant APIs. For interpretation, see text. 
findings, as shown by the rest of Fig. 2, that the apparently equivalent twig branches off independently from the axillary artery, and reinforces the blood supply of this muscle. Typologically considered, it is well understood that this twig passes in front of the Ansa, so far as such is closely akin to the $A$. thoracoacromialis. The authors are of opinion, therefore, that it would represent the rudimentary API, which has failed to develop in such a way as is devoid of the terminal portion reaching the lowest neurovascular hilum of the pectoralis major. In actual fact, this particular hilum receives the direct continuation of the $R$. pectoralis itself, which may be interpreted equivalent to the API.

\section{Discussion}

When the present authors first started the study on the ramification of the axillary artery, their main concern was directed primarily towards a search of convincing peculiarities of the A. subscapularis superficialis. In short, this artery had to be newly defined because it sharply differs from the A. thoracica lateralis, even if the latter standard one, and originates from the artery may appear to be a side branch of the former artery. This is based on the assessment that the $\mathrm{A}$. thoracica lateralis should be one of the most fundamental branches of the axillary artery, and in actual fact, it gives origin to the otherwise deep A. subscapularis.

During the course of this investigation, they have soon realized that from the proximal portion of the axillary artery ramify all the anterior branches, because this artery is, in its first and second parts, lies rather anteriorly to the brachial plexus. It was at this stage that the API, which was then anonymous, has attracted special attention.

So far as the terminal branch entering the lowest neurovascular hilum of the pec- toralis major is concerned, it comes from various sources. It is true that this branch may extend directly from the $R$. pectoralis of the A. thoracoacromialis, passing between the two pectoralis muscles, the major and the minor (Fig. 2). But this is a rare occasion, and the equivalent branch ramifies rather independently, from elsewhere.

Into the anterior muscular cover of the pit of axilla, the $R$. pectoralis is distributed from above the pectoralis minor muscle. But the pectoralis major muscle is usually supplied also by the second branch, which ramifies independently and enters, from below the pectoralis minor, the lowest neurovascular hilum together with the lowest branch of the pectoral nerve. This additional branch varies considerably. It may originate in common with one of the neibouring anterior branches such as the $A$. thoracoacromialis (Fig. 3a, Ser. 2), or the A. thoracica lateralis which includes the $\mathbf{A}$. subscapularis superficialis, a developed system of this primary artery (Ser. 3). But otherwise, it ramifies separately, from that part of the axillary artery, which is located in front of the brachial plexus. If independent, the artery may be divided, from proximal to distal, into three stages as Ser. 1, 4 and 5 (Figs. 3a and 3b), according to its relation to the Ansa. On the same criterion, the combined arteries mentioned above (Ser. 2 and 3) fall into the most proximal group of branches. So far as the present investigation indicates, the most distal artery (Ser. 5) appears to be always independent, but the A. thoracica lateralis may possibly be combined with it, provided it arises from between the Ansa and that of the median nerve, even though such an atypical $\mathbf{A}$. thoracica lateralis rarely happens.

The ramification of the API also varies. Starting from the most simple form (Fig. 3a, Category A), the artery may send off additional branch or branches. They are: the 
muscular branch to the pectoralis minor (Category B), the A. thoracica superficialis in addition (Category $C$ ), and in the end, the A. brachialis superficialis superior (Categories D-F). The last mentioned artery, if present, may far exceed in calibre the API itself, and consequently, the API appears to be an unimportant offshoot of the A. brachialis superficialis.

If attention is paid to the varying origins (Figs. 3a and 3b), it is now clear that the API and the A. brachialis superficialis perfectly coincide with each other on the same line of variation (Ser. 1, 4 and 5). Incidentally, the pattern of transition from categories A-F clearly indicates that the two arteries vary on the same line, being consecutive to each other. Only when the A. brachialis superficialis is poorly developed, impression may be reversed in such a way that this artery appears to ramify from the API (Category D). It should be noted also that every branch of this system is interpreted as superficial. And in addition, it is of particular importance to note that the API always arises superficially, and in no probability from the $A$. brachialis or from any other deep branches of it.

On the other hand, if we pay attention to the branches of the $A$. brachialis superficialis superior, it is noticed that this artery usually gives, most proximally, a muscular branch to the pectoralis major at its lowest neurovascular hilum. Up to the present, practically no attention has been paid to this particular branch, but if one carefully compares the findings with the standard examples, where the A. brachialis superficialis does not exist, he will notice without difficulty that the equivalent artery usually arises independently from the axillary artery. Needless to mention, this is nothing but the API according to the authors' own definition.

Fig. 6 illustrates three types of the A. brachialis superficialis, as the suprema, the

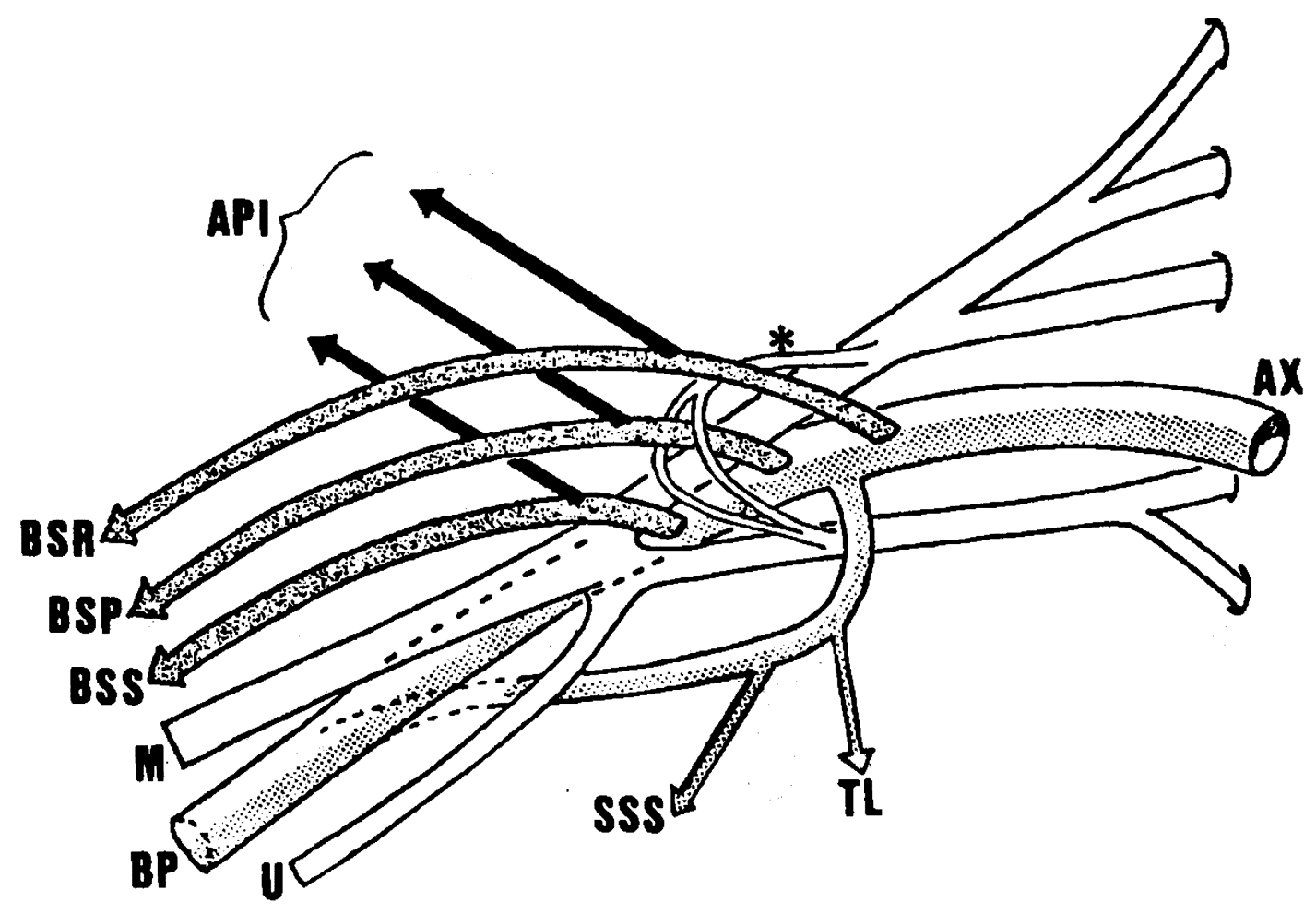

Fig. 6. Relations of API to different A. brachialis superficialis superior (BSR: suprema, BSP: perforans, BSS: superior in a narrow sense). SSS: A. subscapularis superficialis to arise from $A$. thoracica lateralis (TL). U: N. ulnaris. Other abbreviations, see Fig. 1. 
perforans and the superior, each of which is combined with the API of its own. These are the authors' arbitrary definitions, but there is no doubt that these arteries correspond to the A. brachialis superficialis superior of Adachi (1928). But, Adachi and other authors simply neglected the Ansa and its relation to the API. Because of this reason, the API was never received attention before.

In conclusion, the API may be summarised to be an artery from various origins, and is destined to the lowest neurovascular hilum of the pectoralis major. There are alternative routes available to do so, one of which is allotted to the R. pectoralis of the A. thoracoacromialis, and the other, which is almost constant, to the independent APIs arising directly from the axillary artery. The latter is morphogenetically significant because it may extend and gives rise to the superficial trunk artery of the arm. It may be logical, therefore, to divide this superficial system into two portions, the proximal and the distal. Although the conventional description pays very little attention to its proximal portion, it cannot be forgotten that the distal portion would never exist unless the proximal portion paves the way for it. In other words, if any kind of the API is detected anywhere, it would suggest the possible appearance of the superficial trunk artery to extend from there, and to supply whole of the free upper limb.

In such examples where the R. pectoralis is strong enough, the equivalent API becomes weaker and rudimentary (Fig. 2). Thus Fig. 2 (top) would suggest that the API may arise from the distal part of the $A$. thoracoacromialis. In the meantime, the presence of aberrant artery like Fig. 5 would also suggest another possible route for the superficial artery, which branches off from the A. thoracica lateralis, making detour from beneath and round the medial border of the axillary vein.

Finally, the authors are inclined to think that in certain fields of gross anatomy, if not all, re-evaluation should be made before putting our anatomy on the more profound scientific basis. The API may be one of such examples. Despite that the A. brachialis superficialis may completely compensate whole of the artery of the upper limb, Gray's Anatomy by Williams and Warwick (1980) mentions that sometimes the axillary artery divides into the radial and ulnar arteries. To the same phenomenon, Lanz and Wachsmuth (1935) refers as "hohe Teilung" of the brachial artery. Both descriptions may be remarked as superficial because these are likened to a drama which lacks prelude.

\section{Appendix (Glossary)}

1) A. brachialis superficialis suprema (BSR), A. brachialis superficialis perforans (BSP, A. brachialis superficialis superior (narrow sense)(BSS): These three arteries fall into single category of A. brachialis superficialis superior by Adachi (1928) and his predecessors in a broad sense, because they do not take into account the relation of the ansa pectoralis to the artery. But, it may be subdivided as such according to the relation of the ansa. See especially Fig. 6.

2) A. pectoralis inferior (API): Muscular branch which enters the lowest neurovascular hilum of the pectoralis major. For details, see text.

3) A. subscapularis superficialis: A subscapularis is substantially a deep branch which arises from the third part of A. axillaris. But it arises often (ca. 30\%) from A. thoracica lateralis and makes way around the medial cord of the brachial plexus to reach its destination. Of particular interest is that this artery may extend distally to the deep A. brachialis, when A. axillaris of Type $\mathrm{C}$ of Adachi comes into existence. From this interpretation $A$. thoracica lateralis 
is proved to lie on the distal border of the anterior branch. See especially Fig. 6 (SSS). This is the reason why SSS is involved in A. thoracica lateralis.

4) A. thoracodorsalis superficialis: This is an artery on the same line as the preceeding one. The difference is that $\mathbf{A}$. thoracodorsalis, i.e., one of the two major constituents of A. subscapularis, splits off and moves to A. thoracica lateralis. In this instance A. circumflexa scapulae remains deep. This shows that the transition from the deep to the superficial takes place in grades, but is never reversed.

5) A. thoracica superficialis (TS): This is a typical anterior branch of A. axillaris, arising from API, BSR, BSP, BSS or distally even from A. brachialis (as a rudimentary twig equivalent to the A. brach. superf. lat. sive med. inf. of Adachi) (Mizukami, 1966, 1967). Adachi (1928) mentions that it (A. thoracalis superf.) is almost constant. Although it coninues to the $\mathrm{R}$. mammarius lateralis (PNA), PNA is not quite right because the $\mathbf{R}$. mammarius lateralis does not necessarily arise as PNA implies, nor it is by no means specific to females as some authors (e.g., Williams and Warwick, 1980) stress.

\section{References}

1) Adachi, B.: Das Arteriensystem der Japaner, 1.Bd., 210-309, Acta Sch. Med. Univ. Imp. Kioto, IX (1928)

2) Lanz, T. v. and Wachsmuth, W.: Praktische Anatomie, 1/3 Arm, 117-118, Springer, Berlin (1935)

3) Mizukami, S. : R. mammarius lateralis. Unpublished notes circulated among students $(1966,1967)$ (in Japanese)

4) Nishi, S.: Typologische Anatomie des Muskelsystems. Nisshin Igaku 43: 137-145 (1961) (in Japanese)

5) Williams, P.L. and Warwick, R. (eds.): Gray's Anatomy, 36th ed., 700-702, ChurchillLivingstone, Edinburgh, London, Melbourne, New York (1980)

6) Yamada, M.: Significance of the superficial subscapular artery, New designation. Nihon Iji-Shimpo 60: 3-7 (1967) (in Japanese) 